\title{
Enhanced Sampling and Analysis, Selection of Technology for Testing
}

David Meikrantz John Svoboda

February 2010

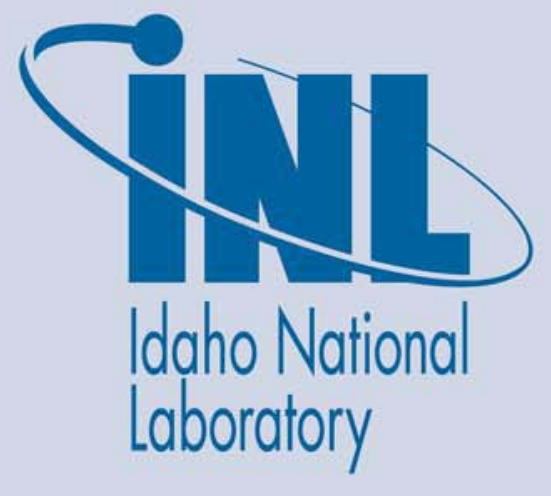

The INL is a U.S. Department of Energy National Laboratory operated by Battelle Energy Alliance 
INL/EXT-10-17919

FCRD-SEPA-2010-000032

\title{
Enhanced Sampling and Analysis, Selection of Technology for Testing
}

\author{
David Meikrantz \\ John Svoboda
}

February 2010

\section{Idaho National Laboratory \\ Fuel Cycle Research \& Development Idaho Falls, Idaho 83415}

http://www.inl.gov

Prepared for the

U.S. Department of Energy

Office of Nuclear Energy

Under DOE Idaho Operations Office

Contract DE-AC07-05ID14517 


\section{DISCLAIMER}

This information was prepared as an account of work sponsored by an agency of the U.S. Government. Neither the U.S. Government nor any agency thereof, nor any of their employees, makes any warranty, expressed or implied, or assumes any legal liability or responsibility for the accuracy, completeness, or usefulness, of any information, apparatus, product, or process disclosed, or represents that its use would not infringe privately owned rights. References herein to any specific commercial product, process, or service by trade name, trade mark, manufacturer, or otherwise, does not necessarily constitute or imply its endorsement, recommendation, or favoring by the U.S. Government or any agency thereof. The views and opinions of authors expressed herein do not necessarily state or reflect those of the U.S. Government or any agency thereof. 


\section{SUMMARY}

The focus of this study includes the investigation of sampling technologies used in industry and their potential application to nuclear fuel processing. The goal is to identify innovative sampling methods using state-of-the-art techniques that could evolve into the next generation sampling and analysis system for metallic elements.

Sampling and analysis of nuclear fuel recycling plant processes is required both to monitor the operations and ensure Safeguards and Security goals are met. In addition, environmental regulations lead to additional samples and analysis to meet licensing requirements. The volume of samples taken by conventional means can restrain productivity while results samples are analyzed, require process holding tanks that are sized to meet analytical issues rather than process issues (and that create a larger facility footprint), or, in some cases, simply overwhelm analytical laboratory capabilities. These issues only grow when process flowsheets propose new separations systems and new byproduct material for transmutation purposes. Novel means of streamlining both sampling and analysis are being evaluated to increase the efficiency while meeting all requirements for information.

Fiscal year 2009 work $^{1}$ provided a preliminary evaluation of potential advanced sampling technologies including an automated microchip capillary based sampling system interfaced to an Inductively Coupled Mass Spectrometer. The ability to obtain micro-liter volume samples coupled with remote automated means of sample tracking and transport to the instrument would greatly improve analytical efficiency while reducing both personnel exposure and radioactive waste. Application of this sampling technique to new types of mass spectrometers for selective elemental isotopic analysis could also provide significant improvements, less time for sampling, analysis, and less waste in safeguards and security analyses.

This report details the selection of an enhanced sampling technology and includes a further consideration of the research focus and goals for this year. The sampling options and focus for the next generation sampling method are presented along with the criteria used for choosing our path forward.

It has been decided to pursue the option of evaluating the feasibility of microcapillary based chips to remotely collect, transfer, track and supply microliters of sample solutions to analytical equipment in support of aqueous processes for used nuclear fuel cycles. Microchip vendors have been screened and a choice made for the development of a suitable microchip design followed by production of samples for evaluation by ANL, LANL, and INL on an independent basis. 


\section{CONTENTS}

SUMMARY iii

ACRONYMS $\mathrm{V}$

1. RESEARCH FOCUS FOR ENHANCED SAMPLING OF REMOTE AQUEOUS PROCESSES 1

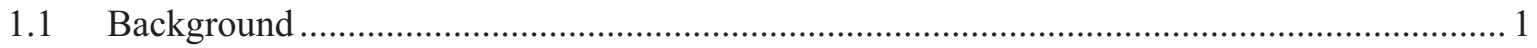

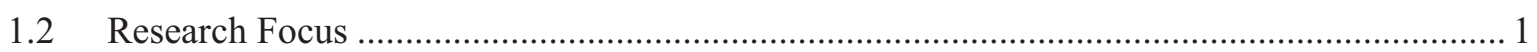

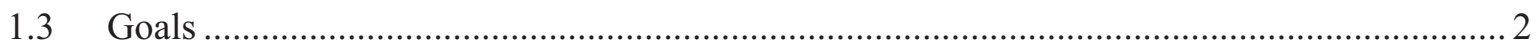

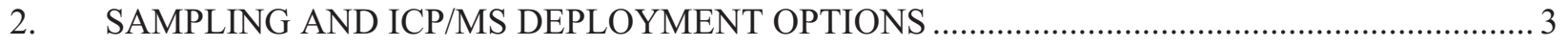

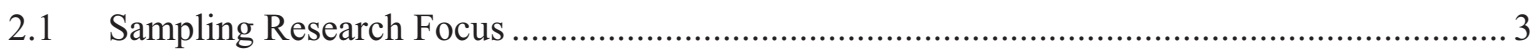

3. VENDOR AND TECHNOLOGY SEARCH FOR MICROFLUIDIC MICROCHIPS .................... 6

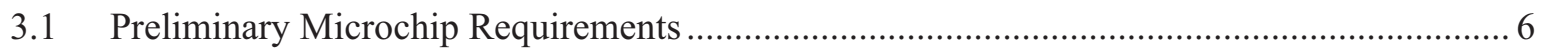

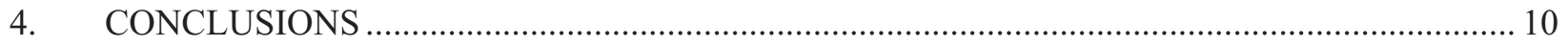

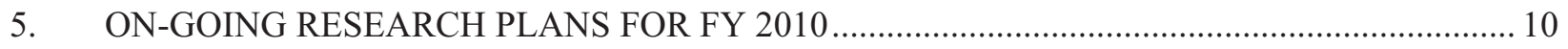

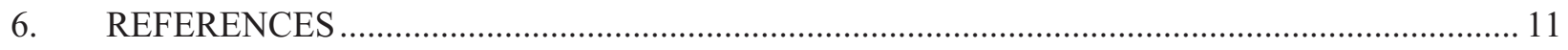

\section{FIGURES}

Figure 1. Capillary Mask vs Channel Width Dimensions.................................................................... 7

Figure 2. Thermally Bonded Capillary With one Etched Surface. ............................................................. 7

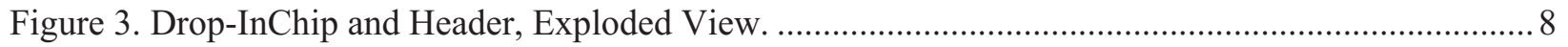

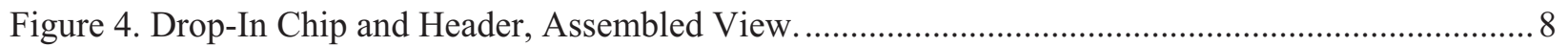

Figure 5: Twelve Fluidic Connection,Disassembled. ............................................................................. 9

Figure 6: Twelve Fluidic Connection, Assembled................................................................................ 


\section{ACRONYMS}

AFCI Advanced Fuel-Cycle Initiative

ANL Argonne National Laboratory

COEX Co-extraction of actinides

FCRD Fuel Cycle R\&D program

IAEA International Atomic Energy Agency

ICP-MS Inductively-coupled mass spectrometer

INL Idaho National Laboratory

LANL Los Alamos National Laboratory

NNSA National Nuclear Security Agency

R\&D Research and development

SNM Special nuclear material

TARIS Thermal atomization resonance ionization spectroscopy

UREX Uranium extraction (includes technetium) 


\section{Enhanced Sampling and Analysis, Selection of Technology for Testing}




\section{ENHANCED SAMPLING AND ANALYSIS, FY 2010 MID-YEAR REPORT}

\section{RESEARCH FOCUS FOR ENHANCED SAMPLING OF REMOTE AQUEOUS PROCESSES}

\subsection{Background}

Within the separations processes, the potential value added results from advanced sampling and analytical methods cannot be ignored. These advances are expected to provide timely analytical results that can minimize facility footprint (process area devoted to storage/surge capacity for analytical turnaround time and analytical laboratory space), ensure timely process control, safeguards, operational safety, and minimized radiation exposure for analytical personnel, and significant reduction in analytical waste generation.

To cost effectively design, build, and operate the next generation of fuel reprocessing facilities, multiple technological challenges require resolution before an optimal facility design and operation scheme can be defined. Facility footprint and timely analytical results are of primary importance and this importance increases with the number of separation systems and separated byproducts that result from flowsheet selection. Research and development that uses a combination of advanced instrumentation, control, and process modeling will eventually be employed to address these concerns:

- A complete understanding of the time/motion characteristics of each sample and its priority (process control, safeguards, safety)

- Identification of techniques that automate sampling and significantly simplify analysis in order to reduce turnaround time

- Identification of on-line monitoring and/or shielded in-line or in-tank analysis that reduce sampling, waste generation and turnaround time

- Integration of automated sample collection with on-line analysis to provide near real time analytical data

- Optimization of sample size to reduce sample waste as well as reduce the time required for the analysis, thereby reducing surge capacity between the major process systems.

\subsection{Research Focus}

This report will focus on the second and last of the above listed concerns as a first step in the effort to provide a transformational method of obtaining, tracking, analyzing, and recording results of aqueous samples taken from remote radioactive processes. Automation will eventually play an important role in this task but first the basic process steps and equipment must be chosen and evaluated.

- The investigation does not assume a separation between analysis technology and sample requirements. The Inductively Coupled Plasma Mass Spectrometry (ICP-MS) is assumed to be the analysis instrument of choice, and it is thought that it can meet most aqueous analysis requirements. The problem, then, is to develop innovative ways to improve the collection of samples, the delivery of samples to the instrument, the analysis process (sample preparation, dilution, injection, cleaning, etc.), and waste management.

- The investigation is primarily constrained to liquid samples because they constitute the majority of the measurements that may be collected from any of the reprocessing paths that are under consideration for example, CO-EXtraction of actinides (COEX), URanium EXtration(UREX), etc. Sampling of gasses and solids will be considered at a later time. 
- In addition, the investigation does not directly address on-line non-destructive sampling technologies because this work is covered by Fuel Cycle R\&D Safeguards and National Nuclear Security Agency (NNSA) funded research.

\subsection{Goals}

The goals of this initial investigation include identifying candidate concepts that can be tested to achieve the following:

- Minimize sample turnaround time from sample extraction and delivery to the analysis instrument to return of analytical data to the intended customer.

- Assure a representative liquid sample at the minimum sample size.

- Minimize waste generation (in the form of sample bottles, analytical lab supplies, decontamination solutions and rinses, sample dilutions, etc.).

- Provide a user-friendly remote sampling by design process that results in, for example, minimal sample points, in-line or in-tank, sampling remote analysis to reduce collection and transport, collocation of remote operated labs within sample stations, etc.

- Minimize worker time required to perform sampling by using automated, as opposed to manual, methods.

- Minimize radiological and chemical exposure of personnel during operation and maintenance.

- Minimize any potential for sample state change during transport, analysis and/or storage (by evaporation, decay, or chemical transitions, etc.). In those instances in which change of state cannot be avoided, characterize/understand the effects of the sample change on resulting measurements.

- Minimize calibration intervals, associated time, and workforce requirements.

- Maximize the reliability and on time availability of sampling equipment.

- Minimize time spent on data management, approvals and record keeping issues. This may be outside our scope unless we put more focus on the turn-around time issues.

- Minimize the uncertainty associated with new liquid sample handling and preparation technology.

- Minimize proliferation risk by reducing sample size and then tracking samples in real time

In addition to these generic sampling goals, the following Safeguards concepts need to be considered and integrated into the R\&D effort:

- Address International Atomic Energy Agency (IAEA) authentication issues so the IAEA can be confident that samples are taken from the stated location and represent the contents of the sampled solution. Examples include:

- Time stamps authenticating the extraction time and the arrival time at the analytic instrument

- Authentication of the location from which the sample was taken

- Authentication of proper homogenization prior to sampling.

- Minimize sample transportation distances, the number of required samples, and the chain of custody.

- Automate, to the extent possible, sampling activities, considering:

- In-line (meaning both sensors and analysis equipment are located in the process flow stream) or on-line (in which the sensors are located in the process flow stream, but analysis equipment is located outside the stream). These two methods are preferred over human involvement in the measurement. 
- Sampling equipment and equipment enclosures incorporate tamper-indicating technology and can be sealed to IAEA standards.

- $\quad$ Time spent on data management, approvals, record keeping and transparency/oversight issues is minimized.

- Measurements by operations personnel are performed by applying a two person rule, including an operator and an IAEA representative. Because this is a labor-intensive and, therefore, expensive way of operating, a better approach will be sought for those circumstances in which a process cannot be fully automated.

\section{SAMPLING AND ICP/MS DEPLOYMENT OPTIONS}

There are three novel methods which might be employed for the analysis of metal ions in nuclear fuel recycling process aqueous streams. It may be feasible to employ specially designed autosampler equipment coupled with remotely employed ICP/MS instruments staged at various locations in the process. Second, the conventional sampling methods that employ shielded sample blisters and stainless steel tubing and needles to recirculate sample solutions from the process to the sample station may be miniaturized via the use of capillary tubing. The last approach to consider is the use of automated equipment to both directly sample and then transfer a microchip based sample to an ICP-MS located outside the radiation zone.

Remotely employed ICP-MS equipment is considered feasible, but would require many expensive instruments and special radiation shielding to protect electronics, and such an instrument would be difficult to maintain, calibrate, and service. Miniaturizing the conventional sampling methods via capillary tubing to reduce sample size, personnel exposure, and waste is troublesome to employ due to the tendency of capillary tubing to fail when solids form from residual solutions due to intermittent sampling. This technique might be useful for monitoring of a flowing stream that is continuously recirculated to the process, but may not adapt well to an ICP-MS. If employable, this method may also require a large number of expensive instruments, one dedicated to each sample point. Thus the third option, the use of automated microchip techniques, appears the most promising and will be the focus of our discussions.

\subsection{Sampling Research Focus}

An entirely new method of acquiring and analyzing radioactive aqueous solution samples during nuclear fuel reprocessing is required to further enhance the recycling of critical energy resources and the life-cycle management of remote handled special nuclear material (SNM), that is, used nuclear fuel. A most promising route for acquiring representative samples appears to lie in the use of automation to gather and transport capillary based microchips. This automated system would acquire the sample directly from the source, track the sample, transport it out of the radiation shielded area, and introduce the sample to an ICP-MS for analysis.

Microchips containing miniscule capillary channels are being employed in a technology commonly called microfluidics. Microfluidics involves the handling and manipulation of minute amounts of fluids, volumes many times smaller than a droplet. The commercial application of microfluidics began almost twenty years ago with the advent of the ink-jet printer. Microfluidics embraces applications in chemistry, biology, medicine, and micro-electromechanical systems. 
Microfluidics is a sea change for liquid handling in the laboratory environment. Most laboratory processes for high throughput bioanalytical screening were based on titer plate technology, parallel processing liquids in wells, each containing up to hundreds of microliters. As titer plates have miniaturized, evaporation of the liquids led to significant analytical accuracy problems. Precise transfer of small volumes of liquids to and from smaller wells also became increasingly difficult. Microfluidics, based on microcapillary chips, provide the basis for solution handling now being applied in biotechnology, drug discovery, chemistry and research in general. A "lab on a chip" is now being employed for electrophoresis based separations using microchip capillary methods for chemical analysis of samples as complex as whole blood. For example, sodium, lithium, magnesium, and calcium can be separated in less than 20 seconds with a lithium detection limit of $0.15 \mathrm{mmol} / \mathrm{L} .^{2}$

This technology, applied to nuclear analytical chemistry, could simplify the accurate determination of important radioelements in the fuel recycling processes. It could begin with the use of microcapillary samplers with automated means of drawing samples from clarified flowing streams and/or well mixed tanks. These chips could contain multiple channels for elemental analyses and simultaneously provide duplicates to ensure precision. Automated transfer of these chips, with bar coding for tracking, could share an interface with spectrometers for both elemental and isotopic analysis. It is probable that instruments could be located inside Perma-Con stainless steel structures to maintain contamination control while remaining available for direct maintenance, calibration, and control because source term for direct radiation levels and related contamination control would be on the order of at least 1000 times lower than today's sample.

Furthermore, the implementation of microfluidics using lab-on-a-chip technology could also provide gains in analytical simplicity, accuracy, and speed. Analytical solvent extraction at the microliter level is possible due to the unique formation of liquid layers when multiple liquids are brought into a single channel. ${ }^{3}$ Reactions and/or mass transfers occur in the vicinity of the interface due to laminar flow. Even miscible fluids form layers in this micro environment, allowing mass transfer in these systems as well.

Methods for remotely sampling representative, but very small amounts (microliters), of the process streams via microchip samplers and demonstrating the compatibility of the sample system with the analytical instruments will be required to take full advantage of this technology. Achieving this goal requires a remote controlled and highly reliable sampling system that can:

- Sample multiple points in the process

- Provide representative samples

- Collect only the volume needed for analysis

- Achieve these tasks in a matter of minutes rather than hours

Microchannel fabrication processes have been demonstrated using timed wet chemical etching methods to selectively etch sacrificial materials. ${ }^{4}$ Prototype glass and polysilicon microchannels thus produced have dimensions of $70 \times 4$ microns and $70 \times 2$ microns, respectively. Both microchannels have been tested to successfully transport and contain water by means of surface tension. A simplified surface tension analysis showed that the height of the etch holes and the surface tension of the working liquid inside the microchannel dominates the pressure difference that holds the liquid from leaking from the etch holes. Because these microchannels are fabricated using standard micromachining processes, the opportunity for economical prototyping and integration with other microfluidic devices is favorable. Further study of the feasibility of adapting and employing microfluidics to nuclear related elemental and isotopic analysis is warranted. Implementing this advanced sampling system on an engineering scale is a key step to assuring successful operations in the next nuclear fuel cycle. 
Automation is becoming more prevalent in analytical, research, and many bioanalytical applications. Autosamplers are being employed for liquid chromatography with the ability to handle samples as small as 0.2 microliters. Fast cycle times of less than 15 seconds for 5 microliter samples are providing reliable, high throughput analyses via such equipment as the Dionex Ultimate 3000 rapid separation autosampler. Such equipment could be adapted for the introduction of very small samples into the ICP-MS or thermal atomization resonance ionization spectroscopy (TARIS) instruments.

The TARIS system potentially could be used to provide an advanced method of single element isotopic analysis for the advanced nuclear fuel recycle facility. TARIS requires a very small sample volume (10 microliters), does not require sample dilution, and can differentiate between radionuclides with the same atomic weight (for example, between ${ }^{238} \mathrm{U}$ and ${ }^{238} \mathrm{Pu}$ ). These advantages would allow the TARIS system to be used to obtain near-real-time analysis of process streams, which will allow accurate tracking of accountable nuclear material throughout the process. However, TARIS requires additional development/verification in order for the system to be implemented into an advanced fuel recycle facility. Further research includes investigation of the ability to analyze all isotopes of interest, automation of the system for multi element analysis, and methods for inputting the samples via microchip technology for direct analysis by the TARIS.

Current ICP-MS instruments have elemental detection sensitivities orders of magnitude lower than those expected for process samples, so dilution of even microliter sized samples will be required. Several dilution methods are being considered to ensure accuracy and reproducibility of the analysis. Therefore, the challenge is focused on how to acquire very small but representative samples, track them at each step, and deliver them to the ICP-MS, reliably. Development of automated elemental separation techniques of samples could also lead to isotopic analysis of required elements by ICP-MS. Implementation of these automated sampling and measurement technologies could provide significant improvements in the efficiency, speed, and accuracy of both Safeguards monitoring and process analysis. Reducing sampling time, sample sizes, analytical time, and direct personnel access will make it possible to collect more data, increase the frequency of sampling, limit personnel exposure, and further reduce risk of proliferation. Additional improvements could include:

- Increased accuracy and decreased measurement uncertainty

- Reduced/optimized process holdup tank sizes for analytical sampling and analysis

- Reduced facility footprint for analytical services

- Reduced facility footprint for holdup tanks for analytical sampling and analysis

- Reduced facility footprint for waste treatment services related to analytical waste

- Optimized operations and decreased down time for analytical results

- Increased frequency of process sampling, significantly lowering the sample volume, and reducing analytical measurement time

- Extended time between process inventory balances via improved accuracy/sample frequency

- Reduced time for analytical measurements, allowing more frequent sampling, thus minimizing the amount of material that can be diverted without detection

- Enhanced process control

- Minimized radioactive waste and sample volume recycle

- Reduced or simplified wet chemical analyses via fewer samples, smaller volumes, less complex sample preparation, and fewer steps

- Secure and reliable data analysis, transmission, output, and tracking. 
February 19, 2010

For these reasons, the decision to pursue the feasibility of employing microchips as the basis for a new sampling system approach was made following the further investigation of the technology and vendors.

\section{VENDOR AND TECHNOLOGY SEARCH FOR MICROFLUIDIC MICROCHIPS}

Off-the-shelf microfluidic products that could be mass produced to meet our sample chip evaluation requirements were initially sought for sample chip investigation. Product lines were examined and found to be tailored to the more complicated problem solutions like chromatography columns, droplet generators, fluid mixing and electrophoresis separations for example. Our need is quite simple, small capillary chips containing a few microliters, compared to the direction of the industry. Initial screening of microchip capabilities pointed to Dolomite Microfluidics who were contacted to design a simple chip specifically for our application and given set of preliminary microchip requirements. Dolomite responded with a proposal that the INL accepted.

\subsection{Preliminary Microchip Requirements}

General: Design a "disposable" microfluidics sample chip used to sample radioactive acidic solutions for elemental metal content using an ICP-MS. The sample chip should be traceable and contain several capillaries such that several samples are obtained on each chip for purposes of redundancy and possibly developing statistics. The capillaries should fill consistently with repeatable volume. Sample chip filling should rely on capillary action. A reproducible sample unloading method should also be considered.

Disposable is defined to mean that they will be cleaned and reused if cleanable. If not, they should be inexpensive so they can be disposed of without incurring high costs.

Sample Solution: Three molar nitric acid solution with 50 to 300 grams per liter of dissolved radioactive heavy metals is the intended use. Design tests may use 50 to 300 grams per liter of a rare earth heavy metal.

Sample Volume: Each sample capillary should contain a volume in the range of 5 to $100 \mathrm{uL}$. The exact volume will be agreed upon after design tests are complete.

Sample Viscosity: Approximately 3 centipoise.

Sample Density: Approximately 1.3 gram/liter.

Sampling method: Each of the capillaries will be filled at the same time by simply touching the solution surface with the sample chip relying on capillary action to fill each capillary tube.

Sample Accuracy/Repeatability: Sample volume repeatability is more important than an actual accuracy. Repeatability estimates will be reported after design tests are complete.

Sample Extraction Method: The sample chip will be placed in an auto sampler based on the chip design. The sample will be removed, diluted with nitric acid and delivered to the nebulizer of an ICP-MS. A header will be connected to the desired capillary of the chip for sample extraction. It should have at least one input and one output tube attached. Ideally a remotely indexed fluidic header would be required that would automatically select the capillary to be analyzed. Design of an automatic sampling system is not part of the scope of this initial work.

Sample Dilution: The sample is expected to be diluted with nitric acid to somewhere between 100:1 at the low end and 10,000:1 at the high end. About $2 \mathrm{~mL}$ of the diluted sample would then be delivered to the ICP-MS nebulizer.

Sample Temperature: The sampled fluid is expected to be between 21 and $45^{\circ} \mathrm{C}$. 
Microchip Fabrication: Approximately 50 or more of the sample chips and about 5 headers will be fabricated such that sets of headers and chips can be sent out for evaluation to all the laboratories. Actual chip numbers and fabrication costs will drive the quantity of headers and chips delivered.

Dolomite Microfluidics: Dolomite Microfluidics was chosen for the design and fabrication. Dolomite is one of the world's leaders in solving Microfluidic problems. Dolomite provides either standard solutions (e.g., chips, interconnects, pumps and valves) or custom designs for devices, sub-systems and instruments. Dolomite has collaborated with several companies, universities, and national laboratories to develop microfluidic solutions for Gas Chromatography, fluid mixing, and microreactions. Dolomite has a worldwide sales presence and a highly motivated design team of engineers and scientists. Capillary masking and chip construction are shown in Figures 1 and 2. Examples of two chips with header variations are shown in Figures 3, 4, 5, and 6.

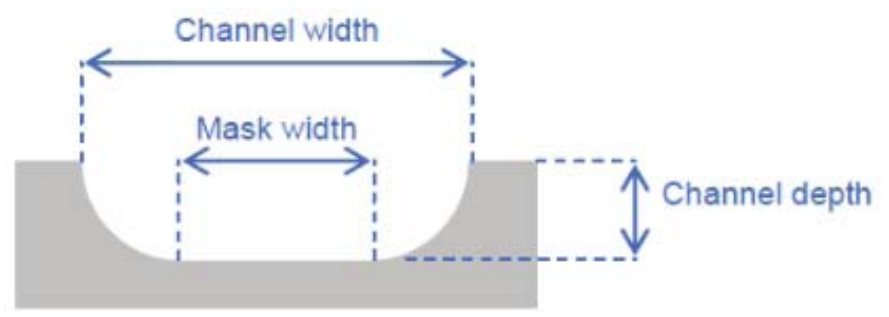

\section{Channel width $=$ Mask width $+2 \times$ Channel depth}

Figure 1. Capillary Mask vs Channel Width Dimensions.

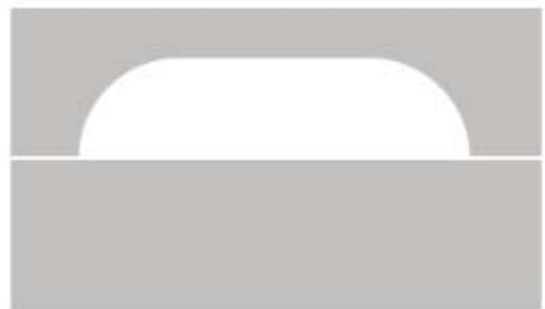

Top glass layer assembled

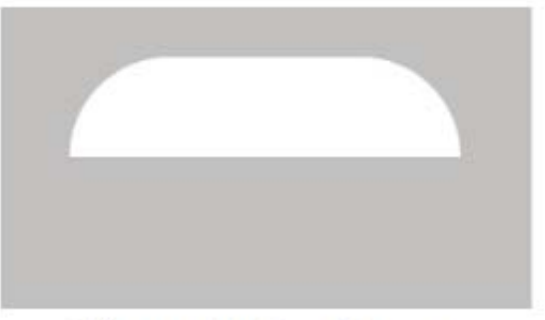

Thermally fuse layers

Figure 2. Thermally Bonded Capillary With one Etched Surface. 


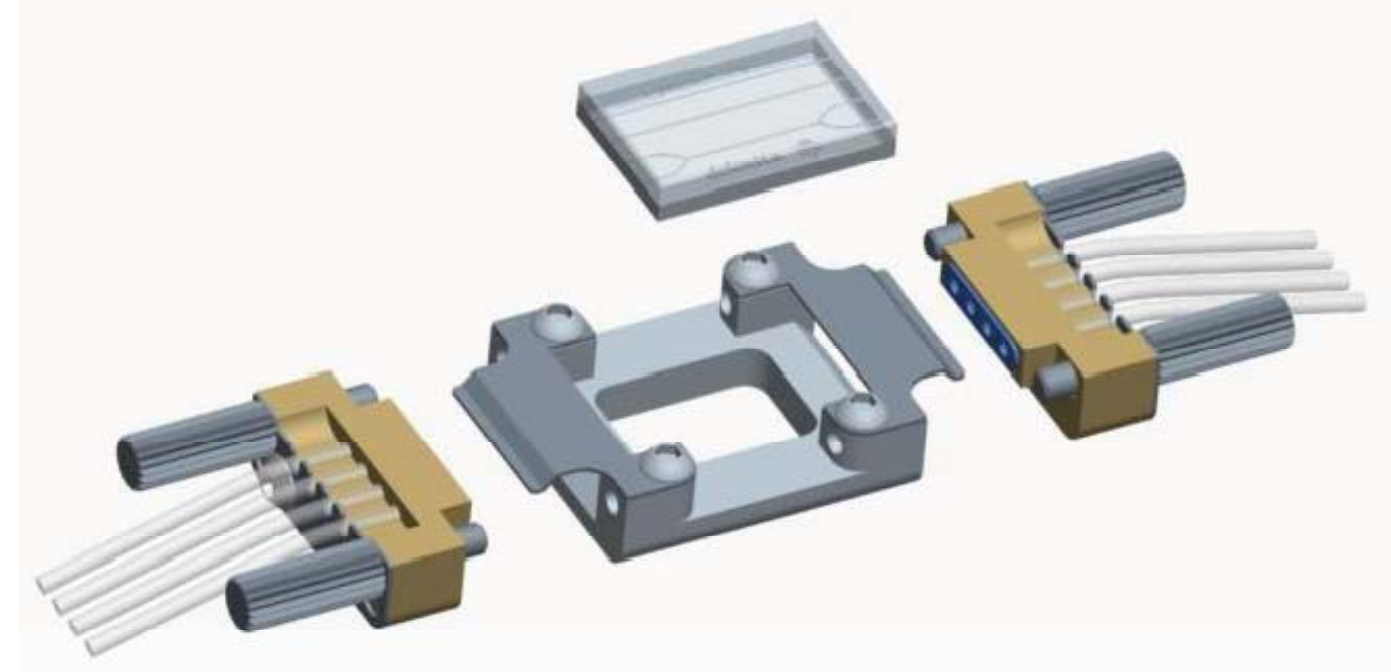

Figure 3. Drop-In Chip and Header, Exploded View.

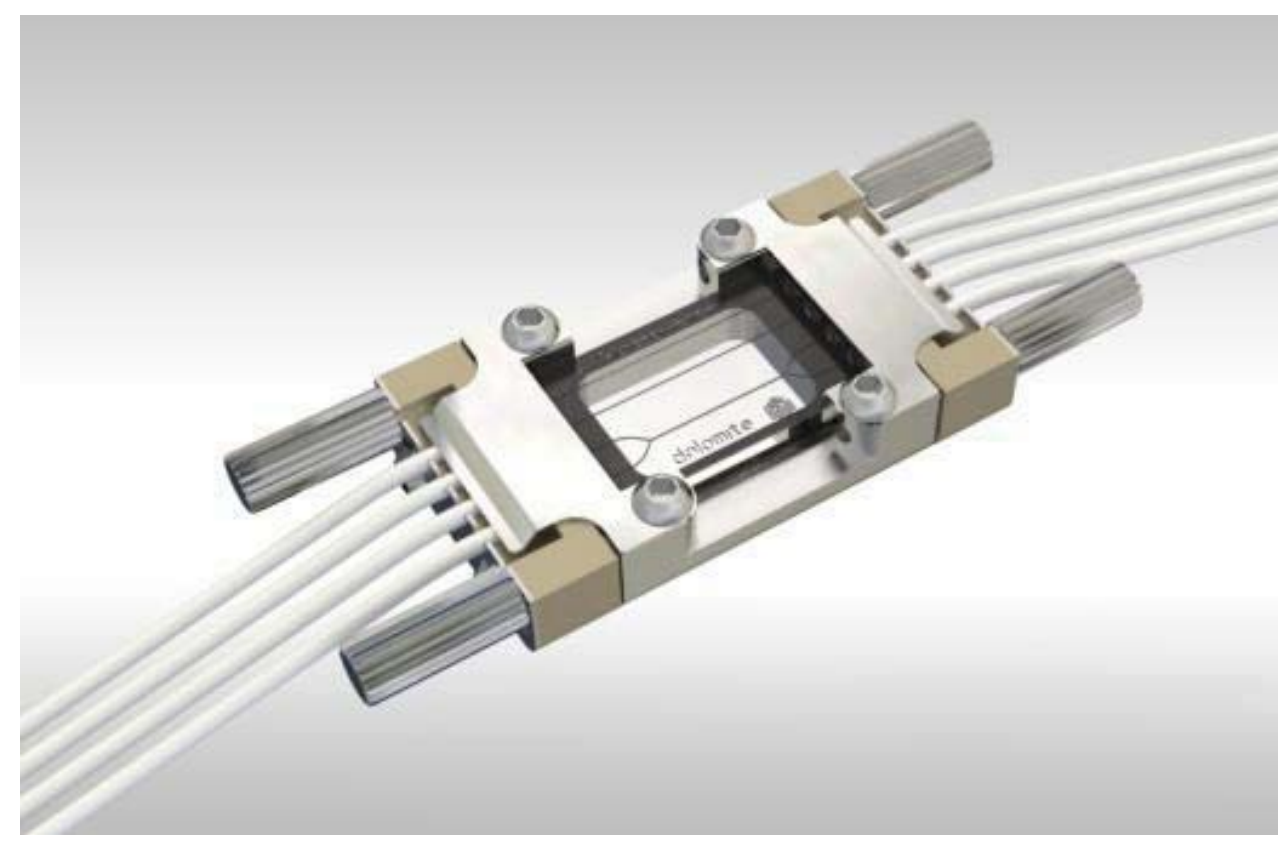

Figure 4. Drop-In Chip and Header, Assembled View. 


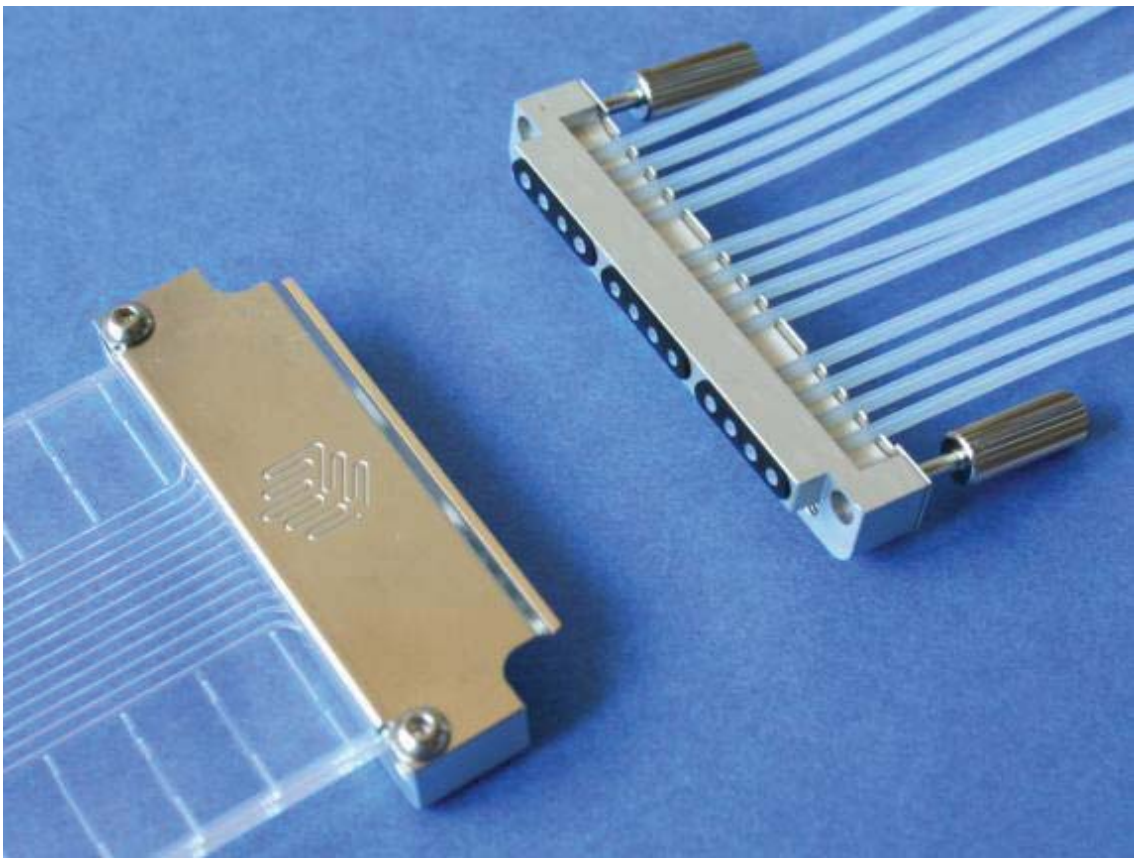

Figure 5: Twelve Fluidic Connection, Disassembled.

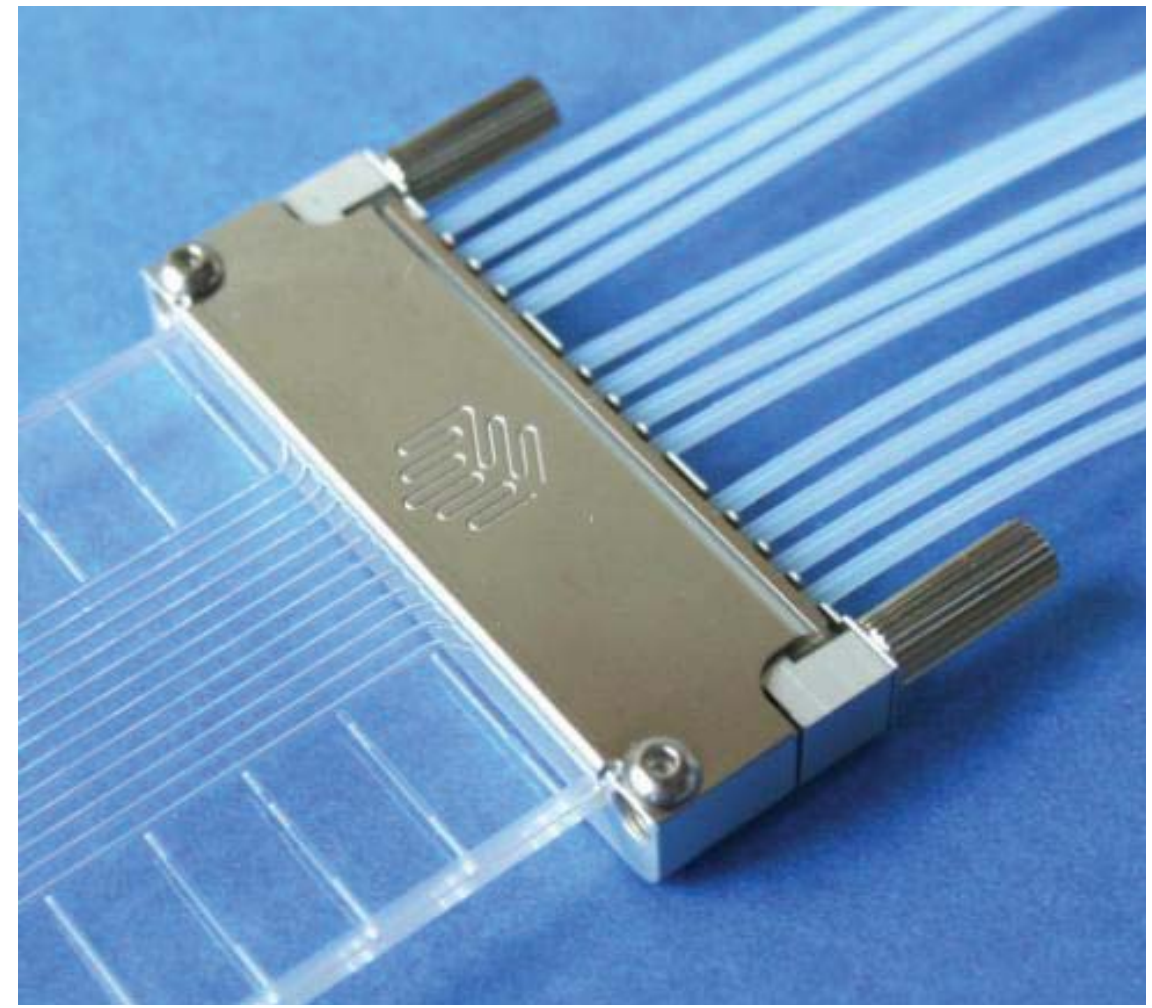

Figure 6: Twelve Fluidic Connection, Assembled. 
Other Dolomite Microfluidic support products include pumps, connectors, valves, flow sensors, and temperature control plates.

Dolomite Proposal: Dolomite generated a proposal that consisted of two phases.

Phase I addresses chip and connector design issues by testing and analysis. Issues to be addressed include droplet attachment to the capillary base, capillary dimensions, evaporation, and header design. Testing will be performed with non-radioactive acidic solutions containing dissolved lanthanides at the target concentrations. A report will be delivered to the INL describing the results. This work is scheduled to take 3 to 4 weeks.

Phase II will create a detailed design based on the outcome of Phase I. The design will be fabricated using $4 \times 4$-in. glass wafers. The number of chips procured will depend on the chip size, number of chips per wafer, and the cost per wafer. Ideally we will procure enough chips and headers to adequately supply the INL, ANL, and LANL with enough to perform the 2010 test series. This phase is scheduled to take 8 to 10 weeks.

\section{CONCLUSIONS}

The most promising new sampling method considered is the use of microcapillary chips and automation to support ICP-MS analysis of the metals needed for both process control and perhaps accountability of SNM in aqueous based nuclear fuel processing. Therefore, we have been in contact with a selected vendor to further the development of this idea. We have initiated steps to establish a non-disclosure agreement to protect and maintain intellectual property created and also a subsequent contract with Dolomite Microfluidics. We have also extended a contract with Advanced Machine Design to support the preliminary concepts and design of a remote sampler device that would directly sample a flowing line or a vessel via the capillary chip method. Through discussions with Dolomite Microfluidics and Advanced Machine Design this process is considered feasible for automation such that a chip can be loaded with sample, tracked and logged via a unique bar code or other marker, and transported to outside the shielded cell area to an ICP-MS for analysis. The sensitivity of the ICP-MS analysis is such that a large dilution of the microliter range sample is required. This dilution factor, on the order of 1000s, will be necessary independent of the method chosen for transferring the sample from the capillary to the analytical instrument input. Therefore, several options for unloading microliter size samples exist, and the actual sample size required is not constrained by the ICP-MS under existing instrument nebulizer input parameters. Dilution of the microliter sample could thus occur via flushing the sample from the capillary using the diluent or dilution following sample removal from the capillary via other means.

\section{ON-GOING RESEARCH PLANS FOR FY 2010}

The remainder of FY 2010 will be spent on the following tasks:

- Optimize the capillary diameter and length to best fit our sampling specifications, verifying the design, and then producing a wafer that can be sectioned into multiple chips for evaluation at INL, ANL, and LANL. This work will be accomplished under contract with Dolomite Microfluidics.

- Perform independent evaluation of the capillary chip samples by each laboratory for suitability as a direct fluid sample method to include:

- Volumetric precision as a function of sample introduction parameters (depth of insertion, angle of insertion)

- Volumetric precision of multiple capillaries in a chip

- Sample stability over expected holding times required for analysis or archive

- Sample stability over expected temperature ranges 
- Sample stability over chip orientation

- Sample stability as a function of concentration

- Sample removal from capillary chip for analysis via factors such as difficulty, method chosen, and analytical sample accuracy.

This task will be shared by INL, ANL, and LANL. Results will be independently reported to the INL lead for documentation and review.

- Consider options for the design of remote sampler devices that could utilize capillary chips to directly obtain samples from solutions in flow lines or tanks using automation. This task shared by INL, ANL, and LANL.

- Produce preliminary designs for employing microfluidic chips as direct samplers. Advanced Machine Design, Dolomite microfluidics.

- Report final results of feasibility of employing microcapillary chip samples in remote nuclear fuel processing applications, recommendations for further development, provide preliminary concept designs for remote sampler, and suggested path forward for developing automation needed for sampling, transport of samples, introduction of samples into ICP-MS, and sample tracking at each step.

\section{REFERENCES}

1. Bowers, D., Henry, R., Law, J., Lamont, S., Meikrantz, D., and Svoboda, J., Enhanced AFCI Sampling, Analysis, and Safeguards Technology Review, INL/EXT09-16997, 2009.

2. Vrouwe, E. X., Luttge, R., Vermes, I., and van den Berg, A., Microchip Capillary Electrophoresis for Point-of-Care Analysis of Lithium, Clinical Chemistry, 53 (1), 117-123, 2007.

3. Hotta, T., Nii, S., Yajima, T., and Kawaizumi, F., Mass Transfer Characteristics of a Microchannel Device of Split Flow Type, Chem. Eng. Technol., 30, (2), 208-213, 2007.

4. Lee, K. B., and Liwei, L. Surface Micromachined Glass and Polysilicon Microchannels using MUMPs for BioMEMS Applications, Sensors and Actuators A: Physical, 111(1), 44-50, 2004. 